\title{
Hierarchical Elastic Graph Matching for Hand Gesture Recognition
}

\author{
Yu-Ting Li and Juan P. Wachs ${ }^{*}$ \\ Department of Industrial Engineering, Purdue University, West Lafayette IN, U.S.A \\ \{yutingli, jpwachs\} @purdue.edu
}

\begin{abstract}
This paper proposes a hierarchical scheme for elastic graph matching hand posture recognition. The hierarchy is expressed in terms of weights assigned to visual features scattered over an elastic graph. The weights in graph's nodes are adapted according to their relative ability to enhance the recognition, and determined using adaptive boosting. A dictionary representing the variability of each gesture class is proposed, in the form of a collection of graphs (a bunch graph). Positions of nodes in the bunch graph are created using three techniques: manually, semi-automatic, and automatically. The recognition results show that the hierarchical weighting on features has significant discriminative power compared to the classic method (uniform weighting). Experimental results also show that the semi-automatically annotation method provides efficient and accurate performance in terms of two performance measures; cost function and accuracy.
\end{abstract}

Keywords: Elastic bunch graph, Graph matching, Feature hierarchy, Hand gesture recognition.

\section{Introduction}

With the growing development of smaller, cheaper and versatile sensors, humancomputer interaction (HCI) relies more on natural communication, as among humans, and less in standard interfaces such as the mice or keyboard [1,2]. This is reflected by the users' subjective satisfaction, the extent of the expressiveness and the overall experience perceived by the users of such systems [3]. Gestures are found extensively as the main channel used to interact with computers in sign language interpretation [2], assistive technologies [4], and game control applications [5]. Recently gestures were adopted in new areas where sterility is essential to the task completion (e.g. browsing medical images in the operating room) [6]. Nevertheless, to make gesture recognition technologies to gain popularity in the HCI common market, high recognition accuracy with low false alarms must be achieved through new pattern recognition techniques.

Elastic graph matching (EGM) is a technique used for object recognition [7], where an object is represented by a labeled graph. The graph is matched against the target image by computing filter responses at each node in the graph, and minimizing

\footnotetext{
"Corresponding author.
} 
a cost function based on some metric applied to the nodes in a template image and a target image. This method was used previously for face and gesture recognition on clutter and complex backgrounds. Elastic Bunch Graph Matching (EBGM) is proposed to recognize the facial images where features were extracted at fiducial points (e.g. the pupils, the nose, and the corners of the mouth) [7]. Triesch et al. employed EBGM to develop a classification approach of hand gestures against complex background [8]. Our improvement with respect to the classic method developed by Triesch et al, consists of assigning an hierarchy to each node in the graph. Those nodes which features are found with higher likelihood on the target image receive a higher hierarchy compared to those nodes which their features are not consistent with the graph model. In other words, nodes with low hierarchy have a reduced effect on the accuracy of the recognition, and thus the matching process can be skipped for them, saving valuable computation time and increasing the accuracy of recognition. Enhancement on the recognition rate is achieved by weighting the linear combination of features in order to assign greater discriminative power to those nodes with higher hierarchies. To summarize, the contribution of this paper are to introduce (1) a hierarchical scheme for elastic graph matching to enhance the hand gesture recognition performance. (2) a study on efficient annotation techniques to create the bunch graph.

The rest of the paper is organized as follows: in Section 2 the EBGM and Adaptive Boosting algorithm are described. Section 3 derives the proposed annotation methods and the hierarchical hand gesture recognition algorithm. Experimental results in Section 4 demonstrate the feasibility and efficiency of the proposed techniques. Finally the discussion and conclusions are presented in Section 5.

\section{Fundamentals of Proposed Algorithms}

\subsection{Elastic Bunch Graph}

Bunch graphs are used to represent and recognize hand posture [8,9]. Each bunch graph is a collection of individual graphs representing a posture. Each node is associated with a vector of complex responses (called jet). The jets are obtained by convolving a set of images (the dictionary set) with a bank of Gabor filters and extracting the responses on specific locations on the images (labeled nodes). The vector of complex responses at a given pixel $\overrightarrow{\boldsymbol{x}}$ follows the form:

$$
\psi_{k}(x)=\frac{\overrightarrow{\boldsymbol{k}}^{2}}{\sigma^{2}} \exp \left(-\frac{\overrightarrow{\boldsymbol{k}}^{2} \overrightarrow{\boldsymbol{x}}^{2}}{2 \sigma^{2}}\right)\left[\exp (i \overrightarrow{\boldsymbol{k}} \overrightarrow{\boldsymbol{x}})-\exp \left(-\frac{\sigma^{2}}{2}\right)\right]
$$

where $\psi_{k}(x)$ is the Gabor-based kernels where the wave vector $\overrightarrow{\boldsymbol{k}}$ describes the variation of spatial frequencies and orientations, which are represented by the index $v \in[0, \ldots, L-1]$ and $\mu \in[0, \ldots, D-1]$. The different values of $\overrightarrow{\boldsymbol{k}}$ are found using:

$$
k_{v \mu}=k_{v}\left(\begin{array}{c}
\cos \phi_{\mu} \\
\sin \phi_{\mu}
\end{array}\right) \text { with } k_{v}=\frac{k_{\max }}{f^{v}}, \phi_{\mu}=\frac{\mu \pi}{D}
$$


where $L$ is the number of frequency levels and $D$ is the number of orientations. These values were chosen according to what was suggested in previous work [8] and since this choice yielded the best performance: $f$ is chosen to be $1 / \sqrt{2}$, and $k_{\max }=$ 1.7. The width of the Gaussian envelope function is $\sigma / k$ with $\sigma=2.5$. The jet is a complex vector consisting of $L \times D$ filter responses and it is defined as $J_{j}=$ $a_{j} \exp \left(i \phi_{j}\right)$. Once the node positions are annotated on the dictionary images, the bunch graph is created by extracting the responses of the filters on those positions (the nodes). The similarity between the bunch graph and target image is used to measure to likelihood of classification of a given image as a gesture. The similarity function in Eq. (3) uses the magnitude and phase of the jet to find a matching score between the bunch graph and the images.

$$
S_{p h a}\left(J, J^{\prime}\right)=\frac{1}{2}\left(1+\frac{\sum_{j} a_{j} a_{j}^{\prime} \cos \left(\phi_{j}-\phi_{j}^{\prime}\right)}{\sqrt{\sum_{j} a_{j}^{2} \sum_{j} a_{j}^{\prime 2}}}\right)
$$

where $a_{j}^{\prime}$ and $\phi_{j}^{\prime}$ is obtained from $J_{j}^{\prime}=a_{j}^{\prime} \exp \left(i \phi_{j}^{\prime}\right)$, the jet from the target image. The phase based similarity in (3) varies rapidly between continuous pixels, resulting in multiple local maxima; thus it is advantageous to have a good initial estimate about the position of the hand within the target image. During the matching process, the best fitting jet is selected according to the maximum similarity score among the bunch. When all the nodes in each bunch are visited and compared, the total score of the matching is given by a linear combination of the scores between the nodes in the bunch graph and the target image. Additionally, total score of the matching considers the distortion of the nodes by introducing the penalty cost to prevent excessive distortion of the graph. Correspondence between the nodes is established automatically according to their relative position on graph. The classification is determined by the maximum score over all the detectors.

\subsection{Adaptive Boosting}

Boosting $[10,11,12]$, is a general machine learning technique used to design and train classifiers by combining a series of weak classifiers to create a strong classifier. This technique was adopted in our posture recognition algorithm to reflect the hierarchy of nodes in the bunch graphs. In boosting technique, a family of weak classifiers forms an additive model:

$$
F(v)=\sum_{m=1}^{M} f(v)
$$

where $f(v)$ denotes a weak detector, $v$ is a feature vector, and $M$ is the number of iterations (or number of weak detectors) to form a strong classifier, $F(v)$. When training the samples, a set of weights is applied to the training samples and they are updated in each iteration. The updates increase the weight of the samples which are misclassified at the current iteration, and decrease the weights of those which were classified correctly. The weights $\omega_{i}=e^{-z_{i} F\left(v_{i}\right)}$ for each training sample $i$ with class 
label $z_{i}$, are defined so the cost of misclassification is minimized by adding a new optimal weak classifier:

$$
\operatorname{argmin}_{f_{m}} \sum_{i=1}^{N} \omega_{i}\left(z_{i}-f_{m}\left(v_{i}\right)\right)^{2}
$$

Upon choosing the weak classifier and added to $F\left(v_{i}\right)$, the estimates are updated: $F\left(v_{i}\right)=F\left(v_{i}\right)+f_{m}\left(v_{i}\right)$. Accordingly, the weights over the samples are updated by:

$$
\omega_{i}=\omega_{i} e^{-z_{i} f_{m}\left(v_{i}\right)}
$$

In this paper, the gentleboost cost function [10] is used to minimize the error (Eq. 5).

\section{Hand Gesture Recognition Methodology}

\subsection{Node Annotation Techniques}

To create the bunch graph described earlier, posture images have to be annotated (their inner nodes and those over the contour (outer) selected). We compare three methods to accomplish this: the manual, semi-automatic and automatic. A single bunch graph consists of six graphs for each posture. To compare the effectiveness of the proposed annotation methods, two metrics are computed and evaluated: (1) Costs entailed to align the nodes. Nodes need to be aligned with respect to their peers within the bunch graph. Relative displacements of the nodes with respect to each other in the different graphs result in an alignment 'cost'.(2) Recognition accuracy given a bunch graph annotated using different approaches.

The difference among these three techniques is the manner on which the nodes are selected within the hand region. For the semi-automatic and automatic methods, first select a graph among six to be the reference (randomly selected). The silhouette of all the six graphs is annotated manually. Once the outer nodes were identified, the interesting points within the hand region are those highly textured regions within the hand, found using a Harris corner detector. For the five remaining graphs, each graph is aligned with respect to the reference graph. The nodes inside the hand region are selected from a larger set of candidates in the following way: apply a linear assignment problem (the formulation is provided in Eq. (7)) to find the points in the current graph that better correspond (with least displacement) to those points in the reference graph.

$$
\begin{gathered}
\min _{z_{i}}\left(\sum_{i=1}^{N_{1} \times N_{2}} z_{i} d_{i}\right) \\
\text { s.t. } \sum_{j=1}^{N_{2}} z_{j}=N_{1}, z_{j}=0 \text { or } 1
\end{gathered}
$$

where $d_{k}=\left\|\left(x_{i}^{1}, y_{i}^{1}\right)-\left(x_{i}^{2}, y_{i}^{2}\right)\right\|$ is the Euclidian distance $\left(i=1 \ldots N_{1}, j=1 \ldots N_{2}\right)$, $\left(x_{i}^{1}, y_{i}^{1}\right)$ is the node of the reference graph, and $\left(x_{i}^{2}, y_{i}^{2}\right)$ is the node of the graph to be matched. The semi-automatic approach allows users to correct manually those points that were detected automatically by subjective observation, while the automatic method does not allow re-placing the nodes. Thus, the automatic approach saves time however can result in greater assignment cost, and thus worse recognition rates. 


\subsection{Hierarchical Weighting on Features}

The standard approach for graph matching equally weights the similarity function; meaning every node has the same importance in the same bunch graph. However, it is well-known that some features of the posture are more dominant than others, in terms of their discriminative power. Thus, this importance is expressed in terms of assigning a weight (hierarchy) to each node to reflect this attribute within the total similarity metric $\hat{S}_{p h a}$. The similarity metric is weighted by the vector $c$ that represents the discriminatory degree of nodes:

$$
\hat{S}_{p h a}=\sum_{k=1}^{K} c_{k} S_{p h a}\left(B^{(k)}, J\left(x^{(k)}\right)\right)
$$

where $B$ is the bunch graph with node index $k$, and $J(x)$ is the jet from target graph taken at node vector $x$. For different postures, the classifiers are trained separately using the adaptive boosting. Positive and negative feature vectors are extracted from each image to form the training set. Figure 1 shows the similarity response of an example image when the metric is computed with and without hierarchy (the bunch graph is scanned over the image with an increment of 4 pixels). In the left image (with hierarchy) the response is more 'focused' than in the right image (without hierarchy). The similarity scores of the entire image are more discriminative when hierarchy is applied over the classifier. This focused response results in fewer local maxima.
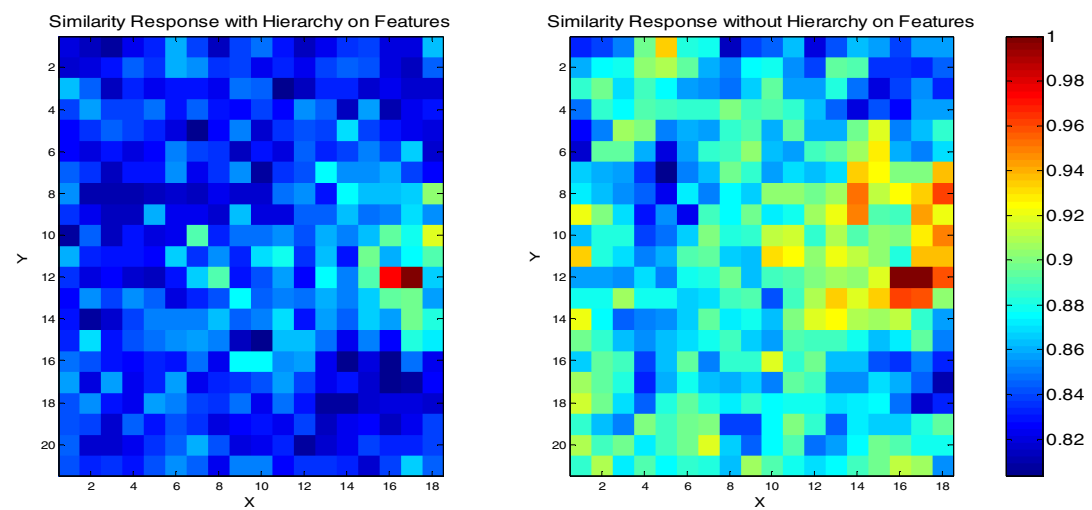

Fig. 1. Similarity response of bunch graph matched to an example image

\section{$4 \quad$ Experimental Results}

The proposed algorithms were validated with the Triesch hand posture dataset [13]. The dataset consists of $730128 \times 128$ grey-scale images with10 different hand gestures against complex, light, and dark backgrounds; performed by 24 people. Each bunch graph is created by selecting two instances of a given posture performed by three subjects against light and dark backgrounds. The geometry of nodes (their position) on the bunch graph is averaged from six graphs. 60 images were used to create the bunch graph. The remaining 650 images were used for the training and testing. The bunch graphs matched on 10 hand postures are presented in Figure 2. 

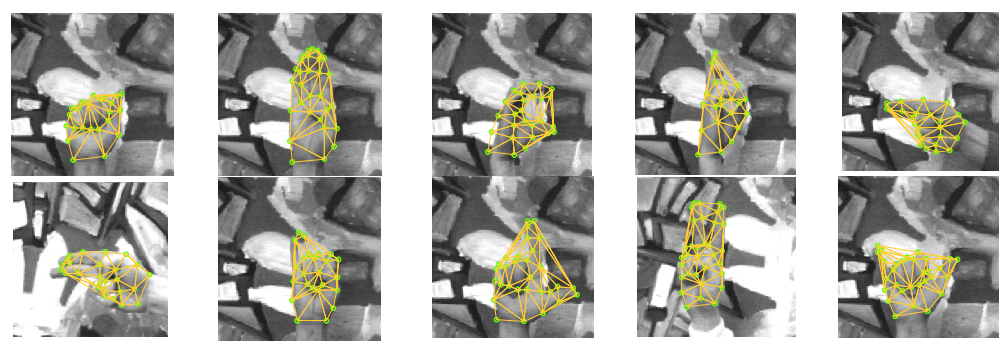

Fig. 2. Ten classes of sample hand gesture images

\subsection{Hand Gesture Classification}

Two metrics are used to evaluate the hand gesture classification performance. First, if the classified score was greater than a threshold (empirically found), then it was regarded as a true positive, otherwise it was a miss. If the negative classes were detected, then those were considered as false alarms. According to these values, a Receiver Operating Characterisic (ROC) curve was plotted to present the relationship between the true positives and false alarms. Figure 3 shows the ROC curves which were generated using 5-fold cross-validation for the 10 hand gestures. The average recognition accuracy was $91.84 \%$.

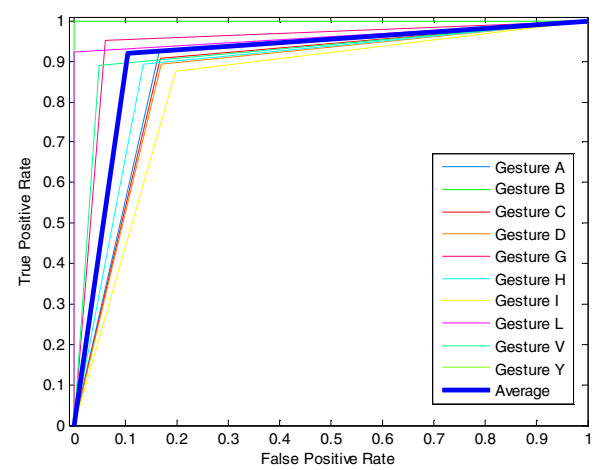

Fig. 3. ROC curve for Hierarchy-based hand gesture recognition

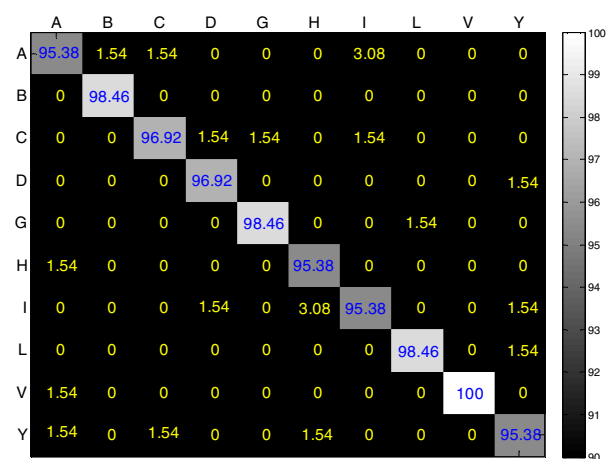

Fig. 4. Confusion Matrix for 10 Gestures 
The second metric was the maximum score over 10 classifiers - this metric always delivered a single detection (correct or incorrect), and no false alarms. If the incorrect gesture was detected, that was considered as confusion. To this end, the confusion matrix (see Figure 4) was obtained by comparing the scores and taking the maximum from all the classifiers applied to the image. The average accuracy of correct classification over the confusion matrix reached $97.08 \%$. Both these values show better performance to those reported in the literature [8].

\subsection{Performance on Different Annotation Techniques}

In this section the performance of each annotation technique used to create the bunch graphs is discussed. Highly textured points inside the hand were detected and considered candidate nodes in the semi-automatic and automatic techniques. The semiautomatic method allowed nodes to be adjusted manually. The results displayed in Figure 5 illustrates that the recognition rate for those classifiers trained using a bunch graph created using a semi-automatic technique tested with light and dark background images $(92.12 \%)$ was better than the other two methods $(90.91 \%$, and $89.26 \%$ for manually and automatically, respectively).The normalized alignment cost was the highest for the automatic technique due to the inconsistency of the nodes' position among the graphs. However, the costs between manual and semi-automatic approaches were comparable. Therefore, the proposed semi-automatic technique shows to be an efficient annotation method for building up the bunch graph.

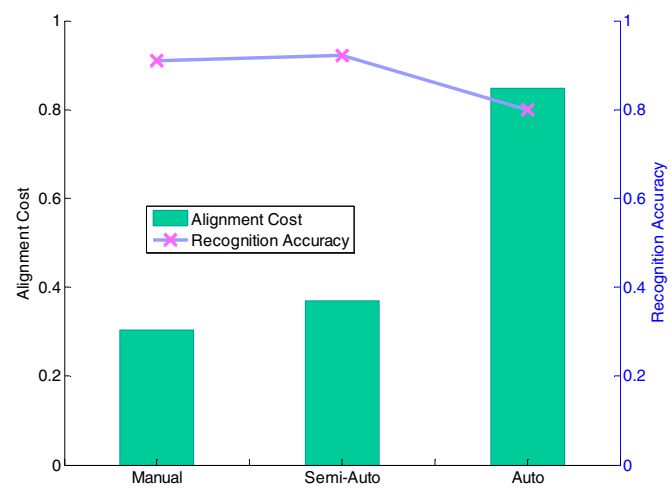

Fig. 5. Alignment cost and recognition accuracy for different annotation techniques

\section{$5 \quad$ Discussions and Conclusions}

This research proposed an enhanced graph based method for hand posture recognition incorporating the concept of nodes hierarchy. The proposed algorithm was tested in a standard dataset of postures using three backgrounds: light, dark and complex. The algorithm suggested classified the postures with a gesture recognition accuracy of $97.08 \%$ on average. This indicates that incorporating hierarchy in the bunch graphs improves the overall performance, since it stressed the discriminatory power of key nodes in every posture. Further work will show a major advantage of hierarchy based 
graphs: they can save computation time; for those nodes with a weight below a threshold, the matching function does not need to be computed.

Another important contribution of this work is a method for fast and reliable annotation of bunch graphs. A semi-automatic annotation technique was proposed which allows the flexible selection of nodes which are consistent between images of the same posture. This method showed to deliver the highest recognition accuracy compared to the manual (traditional) method to construct bunch graphs. Future work will include the validation of this method with a larger and more complex datasets of gestures, and experimenting with different bank of filters other than the Gabor.

\section{References}

1. Poppe, R., Rienks, R., van Dijk, B.: Evaluating the Future of HCI: Challenges for the Evaluation of Emerging Applications. In: Huang, T.S., Nijholt, A., Pantic, M., Pentland, A. (eds.) ICMI/IJCAI Workshops 2007. LNCS (LNAI), vol. 4451, pp. 234-250. Springer, Heidelberg (2007)

2. Roomi, S.M.M., Priya, R.J., Jayalakshmi, H.: Hand Gesture Recognition for HumanComputer Interaction. Journal of Computer Science 6(9), 1002-1007 (2010)

3. Rautaray, S.S., Agrawal, A.: Interaction with virtual game through hand gesture recognition. In: International Conference on Multimedia, Signal Processing and Communication Technologies (IMPACTs), December 17-19, pp. 244-247 (2011)

4. Chang, Y.-J., Chen, S.-F., Chuang, A.-F.: A gesture recognition system to transition autonomously through vocational tasks for individuals with cognitive impairments. Research Developmental Disabilities 32(6), 2064-2068 (2011)

5. Leyvand, T., Meekhof, C., Wei, Y.-C., Sun, J., Guo, B.: Kinect Identity: Technology and Experience. Computer 44(4), 94-96 (2011)

6. Wachs, J.P., Stern, H.I., Edan, Y., Gillam, M., Handler, J., Feied, C., Smith, M.: A gesturebased tool for sterile browsing of radiology images. Journal of the American Medical Informatics Association 15(3), 321-323 (2008)

7. Wiskott, L., Fellous, J.-M., Kruger, N., von der Malsburg, C.: Face recognition by elastic bunch graph matching. In: Int'l Conference on Image Processing, vol. 1, pp. 129-132 (1997)

8. Triesch, J., von der Malsburg, C.: Robust classification of hand postures against complex backgrounds. In: Proceedings of the Second International Conference on Automatic Face and Gesture Recognition, October 14-16, pp. 170-175 (1996)

9. Kumar, P.P., Vadakkepat, P., Poh, L.A.: Graph matching based hand posture recognition using neuro-biologically inspired features. In: 11th International Conference on Control Automation Robotics \& Vision (ICARCV), December 7-10, pp. 1151-1156 (2010)

10. Friedman, J., Hastie, T., Tibshirani, R.: Additive logistic regression: a statistical view of boosting. Annals of Statistics 28(2), 337-374 (2000)

11. Torralba, A., Murphy, K.P., Freeman, W.T.: Sharing features: efficient boosting procedures for multiclass object detection. In: Proceedings of the 2004 IEEE Computer Society Conference on Computer Vision and Pattern Recognition, June 27-July 2, vol. 2, pp. II762-II-769 (2004)

12. Torralba, A., Murphy, K.P., Freeman, W.T.: Sharing Visual Features for Multiclass and Multiview Object Detection. IEEE Transactions on Pattern Analysis and Machine Intelligence 29(5), 854-869 (2007)

13. Sebastien marcel hand posture and gesture datasets: Jochen triesch static hand posture database, http: / /www.idiap.ch/resource/gestures / 